\section{Laser Annealing of Disordered Semiconductors}

\author{
M. Bertolotti, Rome \\ (Institute of Physics, University of Rome)
}

A new and promising application of lasers has grown up over the past 18 months : it concerns laser annealing of disordered semiconductors. Irradiation of materials started soon after the laser was invented but was mainly concerned with effects on metals. Research on semiconductors, motivated by the fact that these materials could be used as mirrors in some laser systems and a knowledge of their behaviour in high laser fluencies would thus be necessary, started somewhat later. Birnbaum in 1965 irradiated germanium with a ruby laser and studied the mechanical effects of the irradiation. A few years later, in 1966-67, workers at the A.F. loffe Physicotechnical Institute of the Academy of Sciences in Leningrad, the P.N. Lebedev Institute of Physics of the Academy of Sciences in Moscow and Moscow University began to study also the effects of laser pulses on free carrier generation and reflectivity. At about the same time, at the Physics Institute of the Engineering Faculty of the University of Rome, we started research on the mechanical damage to many different semiconductors using both optical and electron microscopes, considering also such effects as oxidation and reflectivity changes. The possibility of melting the semiconductor surface and having

Fig. 1 - A spectacular effect of pulsed ruby laser irradiation of a germanium surface. Corresponding to the high energy laser spots, melted material regrows forming conical extrusions [from $M$. Bertolotti et al. J. Appl. Phys. 38 (1967) 4088].

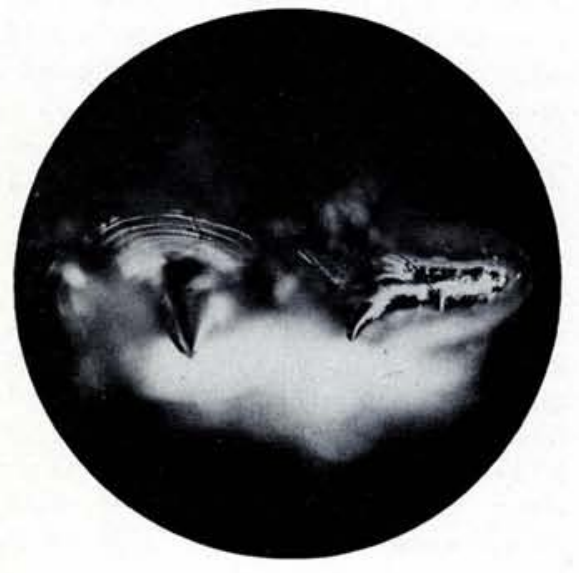

regrowth of the molten material was established.

We had, however, to wait until 1974 before two Soviet groups at the Kazan Physical-Technical Institute and the Institute of Semiconductor Physics in Novosibirsk showed the possibility of recovering electrical properties of ion implanted semiconductor surfaces through laser irradiation.

Ion implantation of semiconductors allows devices to be constructed with carefully controlled characteristics. It consists of bombarding the surface of a semiconductor with ions of some suitable element $(B, P$, In, As, for example) accelerated through potential differences of the order of some tens of kilovolts resulting in a thin region at the surface of the semiconductor (typically 100 to $5000 \AA$ thick) being doped with the bombarding species.

The great advantage of the method over conventional methods, as for example diffusion, is that the ion implanted regions are generally shallower, are formed at lower temperatures, and can be masked by a wider range of materials. Moreover, a much higher concentration of atoms is introduced into the semiconductor than is possible with conventional diffusion techniques.

However, the technique has its drawbacks. The ions introduced in the semiconductor arrive at a rather high velocity and interact with both the electrons and the nuclei of the lattice. In some of the knock-ons between ions and nuclei, the nuclei receive enough energy to be displaced from their regular lattice position, producing disorder and defects. The primarily displaced atoms can also have sufficient energy to displace other nuclei and give rise to defect cascades. Defects can cluster together or can form complexes with some other impurity atoms, as for example oxygen, which is often present. If the bombarding ion dose is high enough, that the implanted region can be considered to have attained a kind of amorphous state. Junctions subject to these effects have poor characteristics. the disorder produced can be so large Berlin - New York

\section{Journal of Non-Equilibrium Thermodynamics}

Editors

J. U. Keller - C. G. Stojanoff

Editorial Advisory Board

B. Baranowski - J. A. Clark . W. E. Falconer - P. Glansdorff -

R. Gregorig · I. Gyarmati .

G. Kelbg · J. Kestin - W. Kollmann A. I. Lopushanskaya - W. J. Moore K. Moritz $\cdot$ W. Muschik - G. Neuer

G. Nicolis - K. Nishikawa -

R. Piccirelli - M. S. Plesset

R. S. Schechter - J. Schroeter -

H. J. V. Tyrrell · M. G. Velarde C. S. Yin

\section{Subscription Information}

1980 , Volume 5 ( 6 issues): DM 285,-; $\$ 175.00$, plus postage and handling.

Single issue: DM 48,-; $\$ 28.00$

Prices are subject to change

Most of the defects can be annealed out by maintaining the semiconductor at a temperature of about $1000^{\circ} \mathrm{C}$ for some hours but in the process the base material can suffer changes or even degradation of its parameters ; the doped impurity may be redistributed and contamination of the surface with uncontrolled impurities can occur.

Fig. 2 - The damage produced at the surface of an ion implanted semiconductor when a low ion dose is confined to some damaged regions (upper part) which at higher bombarding ion doses overlap and produce a more or less uniformly damaged layer (lower part) of nearly amorphous structure.

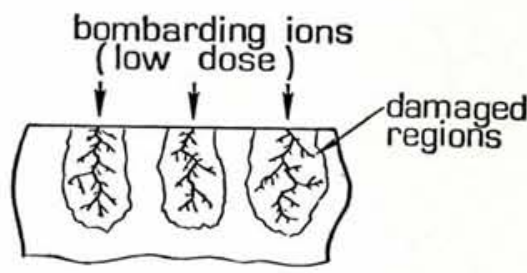

bombarding ions (high dose)

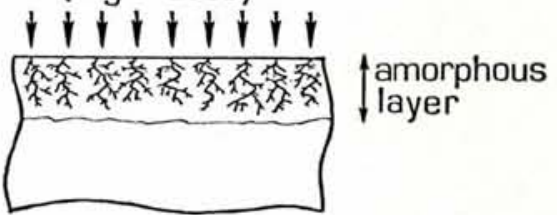


In the same year that the Soviet groups were demonstrating laser annealing, an Italian group decided to look more carefully at the structural changes that occurred under laser irradiation. Carefully implanted $\mathrm{Si}$ samples, doped with silicon ions so as to produce an amorphous surface region of the simplest kind, of different thicknesses were prepared in $\mathrm{Ca}$ tania. They were laser irradiated in Rome with ruby lasers of various characteristics and studied with the electron microscope in Rome and successively in Catania by backscattering. The latter technique depends on the fact that if the bombarding direction is carefully chosen so as to be aligned with one of the open channels in the atomic disposition of the crystal, very few ions are scattered backwards except from atoms displaced from their regular position. The technique is particularly suited to probing the degree of crystalline order under the surface of the sample, whereas the electron diffraction technique is sensitive essentially to the first atomic planes on the surface.

The results of these studies were very exciting. Laser annealing was not only able to restore the electrical properties of the implanted material to their original crystal values but could also produce the following structural changes: amorphous to single crystal, amorphous to polycrystal and fi-

Fig. 4-Backscattering diagram showing the distribution of implanted $\mathrm{Bi}$ in a Si sample as a function of depth under the surface, after laser annealing, compared with the sample as implanted. The curve (black dots) shows that the laser annealing allows the $\mathrm{Bi}$ ions to redistribute themselves in the sample [from C.W. White et al., Appl. Phys. Lett. 33, (1978) 662].

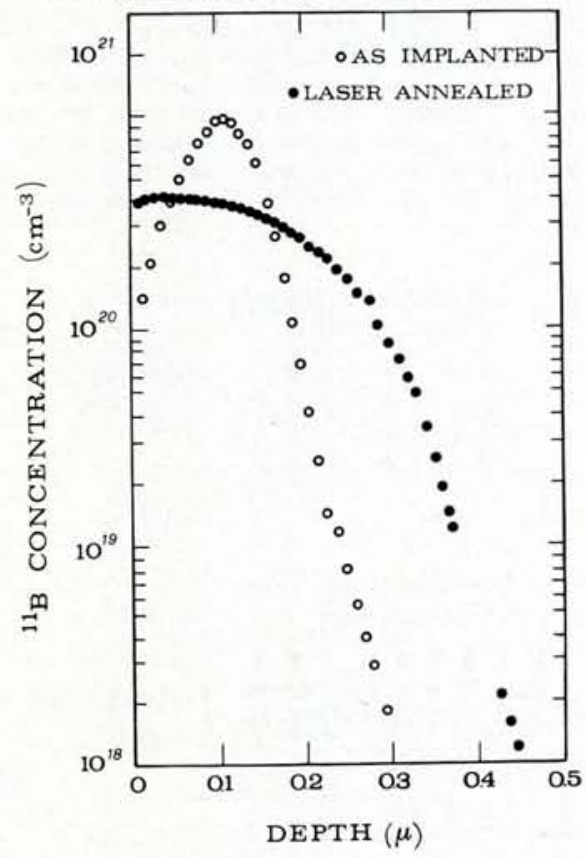

nally polycrystal to single crystal (transition not obtainable thermally).

The mechanisms of the observed effects can be understood as follows :

In the amorphus to single crystal transition under pulsed laser action (for example with a Q-switched ruby or neodymium laser) a thickness of surface material greater than the amorphous layer is melted. Upon regrowing from the single crystal substrate, the material comes up as a single crystal. Moreover, in the liquid state, the dopand atoms may diffuse faster than they would in the solid state, and they redistribute in the sample, reaching nearly ideal distributions.

The amorphous to polycrystalline transition is somehow more involved. It is usually obtained with Q-switched lasers at density powers lower than the values needed to have the singlecrystal transition, and with free generation ruby and $\mathrm{Nd}$ lasers or even with $\mathrm{CW}$ lasers like the $\mathrm{Ar}$ ion laser. It can occur when only a fraction of the amorphous material is melted. In this case the molten material regrows from an amorphous underlying base and only polycrystals are obtained.

In other cases the increase in temperature is not so high as to reach the melting temperature. The material remains, however, sufficiently long at high temperature that the process can take place in the solid phase, as for example, when a CW Ar laser is used or a ruby laser in the free-generation mode. This transition can be obtained also by using free-generation ruby laser pulses in such conditions that the temperature increase is very small. A possible explanation is that a highly-strained situation exists under the laser irradiation which may induce the structural change.

The field of laser annealing is now growing rapidly and promises to become one of the major research areas in the field of ion implantation. In Europe besides the Italian groups mentioned, to which groups in Padova and Modena are now added, research is in progress in England, France, Germany, The Netherlands, Switzerland, USSR. Research started with the effects in $\mathrm{Si}$, but has now rapidly extended to $\mathrm{Ge}, \mathrm{GaAs}$ and other materials, and the possibility of applying it to the fabrication of devices and of ohmic contacts is already under consideration. Laser annealing of disordered semiconductors is a striking example of how fast research can grow once the right direction has been found and how quickly application can follow.

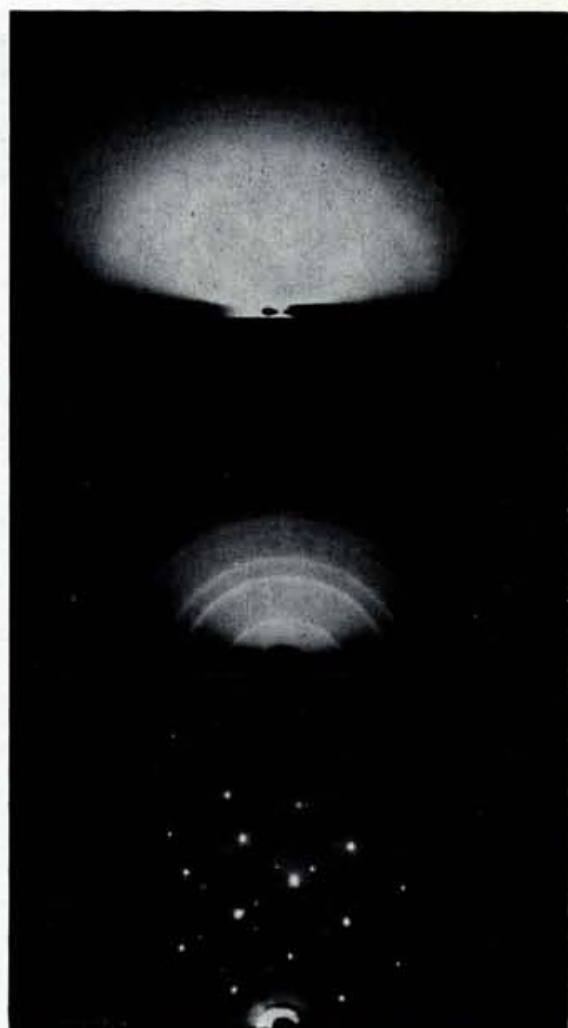

Fig. 3 - High energy electron diffraction patterns showing the different results obtained. From upper to lower: the amorphous Si implanted layer; the same layer after laser irradiation at low power density values giving polycrystalline material (as evidenced by the rings), and the same layer after high power density irradiation showing the formation of a nearlv perfest single crystal (as evidenced by the point pattern) [from G. Vitali et al. Phys. Lett. 63A (1977) 351].

\section{Council's Audience of the Pope on 30 March, 1979}

On the occasion of the meetings held in Rome at the end of March this year, His Holiness the Pope JohnPaul II granted an audience to the EPS Council. In view of the importance of this audience, during which the vital problem of the relationship between Science and the Catholic religion was discussed, and also the interest that has been expressed by a large number of physicists, it has been decided to reproduce the addresses pronounced at that time.

Accordingly, the review Progress in Scientific Culture plans to publish a special issue containing the addresses in their original languages, together with their translations into English, French, German and Russian and some pictures taken during the audience. Copies may be ordered from the undersigned at the price of 5 Sw.Fr. (post-paid). Professor G.-J. Béné, Section de Physique, 24, quai Ernest-Ansermet, $\mathrm{CH}-1211$ Geneva 4. 